\title{
Einführung eines minimalen Datensatzes in den Notfallstationen der Spitäler in der Schweiz
}

Olivier W. Huglia,

Olivier T. Rutschmann ${ }^{b}$,

Roland Bingisser' ${ }^{c}$,

Kaspar Meier ${ }^{d}$

a PD Dr méd., Service des Urgences, Centre Hospitalier Universitaire Vaudois

b PD Dr méd., Service des Urgences, Hôpitaux Universitaires de Genève et Faculté de Médecine de Genève

c Prof. Dr. med., Chefarzt Notfallstation, Universitätsspital Basel

d Dr. med., Chefarzt Anästhesie, Regionalspital Surselva, Ilanz

* Dieses Dokument wurde von den Dres. Olivier Hugli, Olivier Rutschmann und Roland Bingisser sowie Kaspar Meier für die Wissenschaftskommission der SGNOR verfasst

Der minimale Datensatz der Notfallstationen (mDNFS) wurde gemeinsam durch die Wissenschaftskommission der SGNOR und das forum Klinische Notfallmedizin SGNOR in folgender Zusam mensetzung erarbeitet:

Dr. A. Ciurea-Löchel, Bülach Prof. R. Bingisser, Basel PD Dr O. Hugli, Lausanne Dr. H. Matter, Schlieren Dr. K. Meier, Ilanz

PD Dr. J. Osterwalder, St. Gallen PD Dr O. Rutschmann, Genève Dr. M. Schwendinger, Baden

Dr. R. Sieber, Lugano/St. Gallen

Korrespondenz: SGNOR/SSMUS

c/o Gabriela Kaufmann Geschäftsführerin Wattenwylweg 21 CH-3006 Bern Tel. 0313324111 Fax 0313324112

sekretariat@sgnor.ch

\section{Zusammenfassung}

Die Schweizerische Gesellschaft für Notfall- und Rettungsmedizin (SGNOR) empfiehlt, dass die Spitäler für ihre Notfallstationen (NFS) ein standardisiertes Datenerhebungssystem (minimaler Datensatz der Notfallstationen, mDNFS) einführen. Der mDNFS hat zum Ziel

a die Profile der NFS in Bezug auf die Struktur und das Personal zu definieren;

b die Behandlungsprozesse der einzelnen Notfallzentren zu dokumentieren und zu verbessern (Qualitätssicherungsprogramm) sowie Daten für Vergleiche zwischen den Notfallstationen auf schweizerischer oder internationaler Ebene bereitzustellen (Benchmarking);

c mono- oder multizentrische Forschungsprogramme zu erleichtern.

Längerfristig wird die Einführung des mDNFS ein Erfordernis für die Akkreditierung einer NFS in der Schweiz nach den Standards der SGNOR darstellen.

\section{Tätigkeiten der Notfallstationen}

Die jeden Tag rund um die Uhr geöffneten Notfallstationen (NFS) spielen eine entscheidende Rolle in jedem Gesundheitssystem, da sie der gesamten Bevölkerung einen permanenten Zugang zur medizinischen Versorgung für jede Art von Notfall unabhängig vom Schweregrad gewährleisten, ohne dass eine vorgängige Zahlung gefordert wird. Die NFS können somit als Sicherheitsnetz der Gesundheitssysteme betrachtet werden.

Die Inanspruchnahme der schweizerischen NFS hat in den letzten Jahren stark zugenommen, was für alle NFS in den westlichen Ländern gilt [1]. In der Schweiz haben die NFS der Spitäler 2007 beinahe 1,3 Millionen Konsultationen vorgenommen (d. $\mathrm{h}$. rund 17 Konsultationen/100 Einwohner) (Dr O. Hugli et al., unveröffentlichte Daten). Obwohl diese Zahlen deutlich unter jenen der Vereinigten Staaten liegen (120 Millionen Konsultationen, d.h. 40,5 Konsultationen/100 Einwohner [2]), spielen die NFS in der Schweiz eine entscheidende Rolle im Versorgungsnetz, sowohl als Leistungserbringer als auch als epidemiologisches «Observatorium» der Gesundheit der Bevölkerung. Ungeachtet dieser Tatsache liegen in unserem Land praktisch keine Daten zur Tätigkeit der NFS vor.

\section{Bedarf nach einem minimalen Datensatz für die schweizerischen Notfallzentren}

Die Entwicklung einer standardisierten gemeinsamen Datenerhebung oder eines minimalen Datensatzes ist notwendig, da sich damit verschiedene grundlegende Ziele erreichen lassen:

1 Definition des Profils jeder NFS: Gemäss den Empfehlungen der Arbeitsgruppe der Interessengemeinschaft ärztliche Leiter Notfallstationen und der Plattform Rettungswesen FMH $[3,4]$ werden die NFS anhand der folgenden Kriterien definiert:

- strukturelle Merkmale und spezielle Funktionen;

- Zahl und Qualifikation des ärztlichen Personals und des Pflegepersonals;

- demografische Merkmale der Patienten, Verlauf auf der Notfallstation, Diagnosen und Behandlungen.

2. Unterstützung der internen Qualitätssicherungsprojekte durch die Erarbeitung von Indikatoren, die sich auf objektive Daten stützen. Für die NFS wird es auch möglich sein, ihre Leistungen mit internationalen Standards/ Empfehlungen oder mit denen anderer schweizerischer NFS zu vergleichen (Benchmarking).

3. Ermöglichung von epidemiologischen Analysen auf kantonaler oder nationaler Ebene. Da die Kasuistik der einzelnen NFS nur eine fragmentierte Sichtweise ermöglicht, sind Analysen zurzeit schwierig: 2006 lag der Medianwert der Konsultationen pro NFS in der Schweiz bei 9000. Diese Zahl reicht nicht aus, um verlässliche Analysen zu Ereignissen durchzuführen, die nur selten vorkommen. Durch die Zusammenlegung der standardisierten Daten mehrerer Notfallzentren wird es möglich sein, ein eigentliches «Observatorium» für die Bevölkerungsgesundheit einzurichten [5]. Mit diesem Sentinella-Netzwerk lässt sich künftig untersuchen, wie sich die Gewohnheiten im Bereich des Konsums von medizinischen Leistungen im Zusammenhang mit den demografischen Veränderungen entwickeln oder ob neue gesundheitliche Bedrohungen infektiöser, klimatischer oder toxikologischer Art auftreten. So hat der Hitzesommer 2003 gezeigt, dass es hilf- 
reich gewesen wäre, wenn die NFS in der Lage gewesen wären, die Behörden auf die sich abzeichnende Exzessmortalität hinzuweisen [6]. Ein weiteres Beispiel aus der letzten Zeit belegt den Nutzen einer zentralen Datensammlung: 2002-2003 hat die Verwendung eines neuen Imprägniersprays in der Schweiz dazu geführt, dass sechs Patienten die Notfallstation des CHUV wegen einer schweren pulmonalen Erkrankung aufsuchten [7]. Bei diesen wenigen Patienten liess sich zwar vermuten, dass in Verbindung mit der Verwendung der gleichen toxischen Substanz eine toxische Schädigung aufgetreten war. Doch erst die Meldung aller Fälle an eine nationale Institution wie das Tox-Zentrum ermöglichte es, das Ausmass des Problems zu erkennen (über 100 Fälle) und die Gesundheitsbehörden zu alarmieren, damit der Spray vom Markt genommen werden konnte [8].

4. Förderung von Forschungsprojekten: Auch in diesem Bereich verhindert die geringe Zahl von Konsultationen pro NFS, dass die erforderliche Kasuistik vorliegt, um hochstehende Forschungsprotokolle durchzuführen. Die Erhebung von standardisierten Daten sollte auch die Lancierung von prospektiven oder retrospektiven multizentrischen Projekten erleichtern.

Die Wissenschaftskommission der SGNOR - in Zusammenarbeit mit dem forum Klinische Notfallmedizin SGNOR - hat einen minimalen Datensatz für die Notfallstationen (mDNFS) erarbeitet, dessen Rubriken sich auf die folgenden Quellen stützen:

- Formular zur Erhebung von minimalen Daten der Schweizerischen Gesellschaft für Intensivmedizin, Version 22_0_fr [9];

- vom CDC durchgeführte jährliche Erhebung bei den Notfallzentren der Spitäler in den USA [10];

- von Dr. K. Meier geleistete Vorarbeit und Weiterentwicklung im Rahmen von Vernehmlassungen durch Mitglieder des forums Klinische Notfallmedizin SGNOR.

Das mDNFS ist in einem Anhang festgelegt, der auf der Website der SGNOR abgerufen werden kann (www. sgnor $\rightarrow$ Downloads / Klinische Notfallmedizin [Minimal Data Set]). Die Erhebung der Daten soll so weit als möglich automatisch erfolgen: entweder aus dem administrativen Informatiksystem des Spitals und/oder dem elektronischen System zur Steuerung der Patientenflüsse und/oder der elektronischen Krankengeschichte des Patienten und/oder dem System für die elektronische Verordnung. Gemäss einer landesweiten Umfrage verfügten 2007 62\% der NFS über ein elektronisches System, das Daten in Echtzeit bereitstellt. Allerdings ist der Inhalt dieser Informationen je nach Zentrum sehr unterschiedlich (Dr O. Hugli et al., unveröffentlichte Daten). Es wird von grundlegender Bedeutung sein, die entsprechenden Computerprogramme bei ihrer Aktualisierung anzupassen, damit der Datensatz erhoben werden kann.

\section{Empfehlungen der Schweizerischen Gesell- schaft für Notfall- und Rettungsmedizin (SGNOR)}

Auf Antrag ihrer Wissenschaftskommission gibt die SGNOR die folgenden Empfehlungen hinsichtlich der Einführung des mDNFS ab:

1. Jede Notfallstation sollte die notwendigen Daten für einen reibungslosen Betrieb erheben und dabei möglichst von den Rubriken ausgehen, die im mDNFS festgelegt sind.

2. Es steht jeder NFS frei, die Gesamtheit oder nur einen Teil der Daten zu erheben, entsprechend den Ressourcen ihres Personals oder Sekretariats oder ihrem Informatiksystem.

3. Die Wahl des Erfassungsinstruments wird der einzelnen Institution überlassen, wobei das Format zu berücksichtigen ist, das im Dokument im Anhang festgelegt ist.

4. Bei der Weiterentwicklung der spitaleigenen Informatiktools sollte die Erfassung der Daten des mDNFS berücksichtigt werden.

5. Jede NFS legt eine Person fest, die für die Einführung und Betreuung der Datenerhebung verantwortlich ist. Die Personalien werden der SGNOR mitgeteilt.

6. Der mDNFS wird von der Wissenschaftskommission der SGNOR regelmässig überarbeitet, damit es an die Entwicklungen der Berufspraxis und -politik angepasst werden kann.

7. Mittelfristig ist das Ziel anzustreben, dass die anonymisierten Daten an eine zentrale Datenbank weitergeleitet werden. Dieses Ziel wird mit den Notfallzentren noch weiter diskutiert.

Die Literaturhinweise [1]-[10] finden sich in der OnlineAusgabe der SÄZ unter www.saez.ch $\rightarrow$ Archiv $\rightarrow 2011$ $\rightarrow 40$. 


\section{Literatur}

1. Santos-Eggimann B. Increasing use of the emergency department in a Swiss hospital: observational study based on measures of the severity of cases. BMJ. May 18 2002;324(7347):1186-1187.

2. Pitts SR, Niska RW, Xu J, Burt CW. National Hospital Ambulatory Medical Care Survey: 2006 emergency department summary. Natl Health Stat Report. Aug 6 2008;7:1-38.

3. Arbeitsgruppe der Interessengemeinschaft ärztliche Leiter Notfallstationen. Notfallstationen: strukturelle und organisatorische Empfehlungen für die Qualitätssicherung. Schweiz Ärztezeitung. 2005;86:1918-1928.

4. Plattform Rettungswesen FMH. Kategorien von Notfallstationen. Schweiz Ärztezeitung. 2005;86:1915-1917.

5. Claessens Y, Kierzek G, Josseran L, André S, Bernas F, Pourriat J. Services d'urgence: observatoires de santé publique. Réanimation. 2008;17(8):807-815.
6. Claessens YE, Taupin P, Kierzek G, et al. How emergency departments might alert for prehospital heat-related excess mortality? Crit Care. 2006;10(6):R156.

7. Heinzer R, Ribordy V, Kuzoe B, Lazor R, Fitting JW. Recurrence of acute respiratory failure following use of waterproofing sprays. Thorax. Jun 2004;59(6):541-542.

8. Vernez D, Bruzzi R, Kupferschmidt H, De-Batz A, Droz P, Lazor R. Acute respiratory syndrome after inhalation of waterproofing sprays: a posteriori exposure-response assessment in 102 cases. J Occup Environ Hyg. May 2006;3(5):250-261.

9. Kommission Patientenklassifizierung (KPK),SGI-SSMI. MDSi - The minimal dataset of the Swiss Society of Intensive Care Medicine (SSICM). http://www. sgi-ssmi.ch/sgi-mdsi.html?\&L=3. Accessed 14. Juni 2010.

10. Center for Disease Control and Prevention. National hospital ambulatory medical care survey 2008 emergency department patient record http://www. cdc.gov/nchs/data/ahcd/nhamcs100ed_2008.pdf. Accessed 14. Juni 2010. 